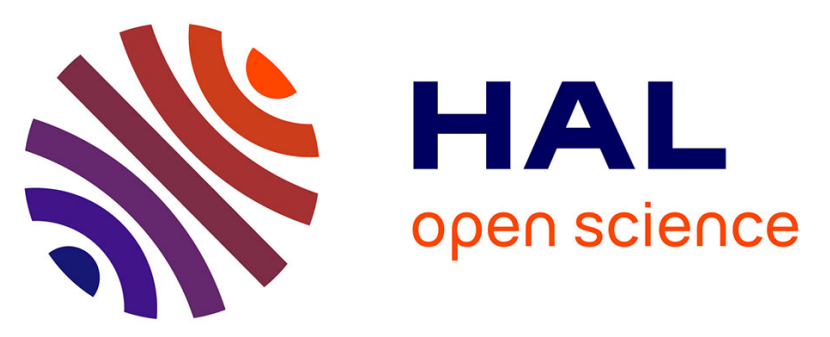

\title{
Microsensors and image processing for single oocyte qualification: toward multiparametric determination of the best time for fertilization
}

Bruno Wacogne, I. Ivascu, Rabah Zeggari, Christian Pieralli, Caroline Amiot, Lionel Pazart, Christophe Roux

\section{To cite this version:}

Bruno Wacogne, I. Ivascu, Rabah Zeggari, Christian Pieralli, Caroline Amiot, et al.. Microsensors and image processing for single oocyte qualification: toward multiparametric determination of the best time for fertilization. Laser Physics Letters, 2013, 10, pp.105601. 10.1088/1612-2011/10/10/105601 . hal-00878479

\section{HAL Id: hal-00878479 \\ https://hal.science/hal-00878479}

Submitted on 30 Oct 2013

HAL is a multi-disciplinary open access archive for the deposit and dissemination of scientific research documents, whether they are published or not. The documents may come from teaching and research institutions in France or abroad, or from public or private research centers.
L'archive ouverte pluridisciplinaire $\mathbf{H A L}$, est destinée au dépôt et à la diffusion de documents scientifiques de niveau recherche, publiés ou non, émanant des établissements d'enseignement et de recherche français ou étrangers, des laboratoires publics ou privés. 


\title{
Microsensors and image processing for single oocyte qualification: towards multiparametric determination of the best time for fertilization
}

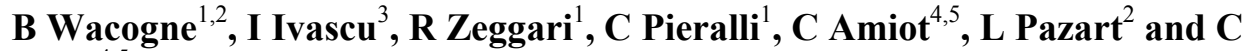 \\ Roux $^{4,5}$ \\ ${ }^{1}$ FEMTO-ST Institute, UMR CNRS 6174, 25000 Besançon, France \\ ${ }^{2}$ INSERM CIT 808, Besançon University Hospital, 25000 Besançon, France \\ ${ }^{3}$ Physics Department, Faculty of Applied Sciences, University "Politehnica" of \\ Bucharest, Splaiul Independentei 313, Bucharest, 060042, Romania \\ ${ }^{4}$ Service de Génétique Biologique, Histologie Biologie du Développement et de la \\ Reproduction, Besançon University Hospital, 25000 Besançon, France \\ ${ }^{5}$ INSERM CBT 506, UMR1098, Besançon University Hospital, 25000 Besançon, \\ France
}

Contact E-mail: bruno.wacogne@univ-fcomte.fr

\begin{abstract}
During Intracytoplasmic Sperm Injection (ICSI) attempts, oocytes reaching metaphase II are microinjected. A morphological examination under a microscope is the usual method for determining oocyte maturity. The level of oocyte maturity is based on the meiotic status (Germinal Vesicle, metaphase I and metaphase II) of the oocytes with respect to their increasing maturity. In this paper, we summarize the studies conducted to analyze cytoplasm maturity using various microsystems and image processing. Optical microsystems are used to measure the transmission spectra and refractive index of the oocytes. We compared the transmission spectra measurements to the transmission electron microscopy results. KarhunenLoeve Transform is also used to evaluate the maturity of the oocytes. To summarize, optical analysis techniques are a minimally invasive technology allowing cytoplasm maturity to be assessed. Oocytes should not only be qualified in terms of GV, MI or MII, but also regarding their temporal evolution over the course of these maturation stages. The ultimate aim of this work is to describe the maturation of the oocytes by a trajectory in a multidimensional space and to determine when would be the best time for successful fertilization.
\end{abstract}

\section{Introduction}

Knowledge of the level of development (from growth to apoptosis) of cells is useful in biology and medicine. This question is even more crucial in IVF (in vitro fertilization), because women undergo intensive hormonal treatment and intervention is expensive. Practitioners must therefore be sure that the oocyte(s) to be implanted is/are the best candidate(s). Sometimes there is no doubt about the level of maturity of an oocyte, but often the practitioner cannot be certain, hence the purpose of our research. There are several degrees of oocyte maturity. In this paper we will focus on three stages of maturity: GV (germinal vesicle), metaphase I and metaphase II.

Since the dawn of IVF, a few technological developments have been investigated. They mainly concern the preparation, conditioning and conservation of gametes, as well as culture of the oocytes and embryos [1]. Some publications report innovation in the technological domain. Some of this innovation concerns the preparation, conditioning and manipulation of oocytes in microfluidic systems [2-7]. Other techniques have been developed to estimate the quality of semen [8]. Oocyte maturity has also been investigated by means of mechanical measurements, in particular for animal oocytes [9-12]. It should be noted that, to our knowledge, only one conference [13] and one paper [14] have reported experiments on human oocytes. The invasive or non-invasive nature of the techniques is probably an important issue. Concerning non-invasive techniques, optical methods offer potential possibilities. 
These techniques have been tested on living cells [15-16], on oocytes [6, 17-19] or on the embryos culture medium [20].

In this study, various techniques for assessing oocyte maturity and, more precisely, the temporal evolution of their maturity are summarized. In the next section, we present the results obtained with a simple optical microsystem used to measure both the transmission spectra of the oocytes and their refractive index $[21,22]$. We also compared the transmission spectra measurements to transmission electron microscopy results [23]. We observed that the evolution of the spectra is consistent with the migration of cortical granules toward the plasma membrane and the reduction of vesicles and vacuoles in the center of the ooplasma. Section three is devoted to the assessment of oocyte maturity by means of the Karhunen-Loewe Transform [17]. After a short discussion of the potential of minimally invasive techniques for assessing the temporal evolution of the maturity of the oocytes in section four, this paper will make conclusions.

\section{A simple optical microsystem}

\subsection{Description of the microsystem}

A schematic diagram and microscope views of the device are shown in figure 1 [24]. The device consists of a micro-machined piece of silicon anodic bonded onto a glass substrate. Only the silicon part of the device is micro-machined. The main features are two U-grooves into which optical fibers are stuck. In this way, optical fibers are perfectly aligned. A specific pigtailing arrangement and fiber stops were designed in order to adjust the distance between the two fibers precisely $(500 \mu \mathrm{m})$ [6]. The core of one fiber is $50 \mu \mathrm{m}$ in diameter. This is the illumination fiber. The core of the second fiber (the collecting one) is $100 \mu \mathrm{m}$ in diameter.

The space between the two fibers is used to position the oocytes manually by means of the micromanipulator arrangement used in IVF centers. The glass substrate allows the oocytes to be positioned precisely with a microscope. Figure 1(b) shows microscope views of the device when an oocyte is placed in front of the illumination fiber.

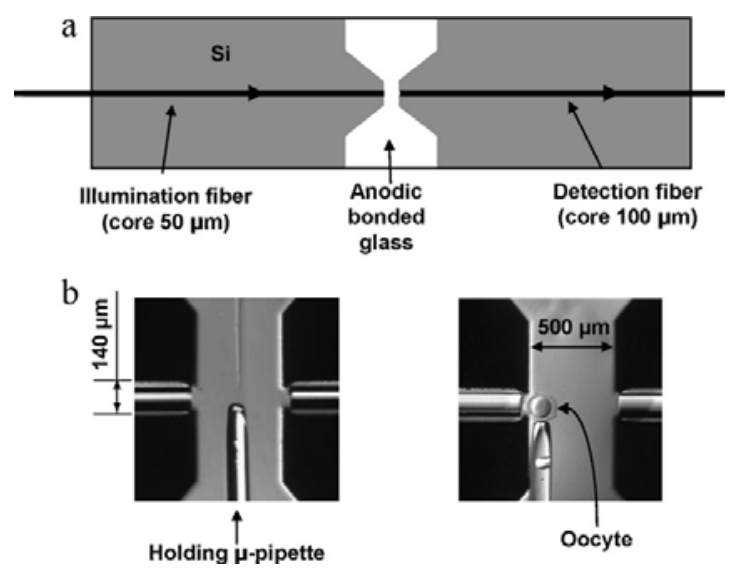

Figure 1. Schematic diagram (a) and microscope views (b) of the device [24].

A low power white light source is used to illuminate the oocytes (Ocean Optics LS-1). The light propagated through the oocytes is then launched into the collection fiber and directed to a microspectrometer (Ocean Optics USB 2000+). In this way, the transmission spectra of the oocytes are easily recorded and the acquisition time can be reduced to $3 \mathrm{~ms}$.

\subsection{Oocyte preparation}


The oocytes used in this study were excluded from Assisted Reproductive Technology (ART) programs. Use of the oocytes was approved by the clinical ethics committee of Besancon University Hospital on 5 May 2004.

The preparation protocol can be found in detail in reference [24]. Oocytes are collected during ART attempts or from follicular reduction. The oocyte cumulus complexes are individualized under a binocular microscope and transferred into a culture medium. After 90 to $120 \mathrm{~min}$ of in vitro culture at $37{ }^{\circ} \mathrm{C}$, the cumulus is removed. Oocytes are individually transferred into a 4 wells petri dish with 100 $\mu 1$ of culture medium. Their level of maturity is defined by visual inspection with an inverted microscope.

Two hundred $\mu$ of IVF culture medium is deposited between the two fibers of the device. One oocyte is collected in the plastic box in $20 \mu \mathrm{l}$ of IVF medium and positioned against the emission fiber. The holding micropipette is employed in order to position the oocyte precisely. Once the measurements are made, the oocyte is put back into the IVF medium in the petri dish. They are then incubated in a Sanyo $\mathrm{CO}_{2}$ incubator $\left(35^{\circ} \mathrm{C}, 5 \% \mathrm{CO}_{2}\right)$.

\subsection{Experimental results concerning the absorption spectra}

Theoretical considerations as well as the detailed experimental procedure are given in [24]. We summarize the main results as follows.

First, we measured the average spectra corresponding to the three maturation stages. Average values were obtained for $57 \mathrm{GV}, 70 \mathrm{MI}$ and $40 \mathrm{MII}$ respectively. We can see in figure 2 (a) that both values of the minimum transmissions and the corresponding wavelengths vary according to maturity. The minimum transmissions increase from 0.34 (a.u.) for $\mathrm{GV}$, to 0.43 (a.u.) for MI and to 0.49 (a.u.) for MII oocytes. At the same time, the wavelengths corresponding to these minima decrease from 464 $\mathrm{nm}$ for $\mathrm{GV}$, to 451 for MI and to 439 for MII oocytes.

Figure 2(b) shows the Box and Whiskers diagram for the three oocyte populations. This diagram allows a particular characteristic (transmission spectra in this case) of different-sized populations to be compared. We can clearly see from this figure that the three maturation stages are well identified. At the same time, it is also obvious that these three stages overlap. This means that, although the stages are identified, the analysis of the transmission spectra cannot be used in a blind test. We will discuss this aspect later.

(a)

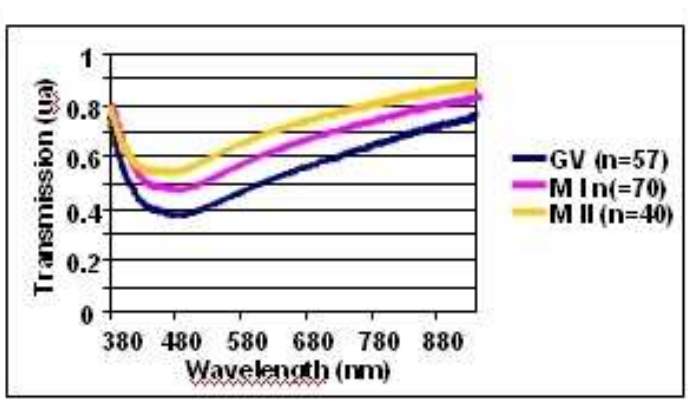

(b)

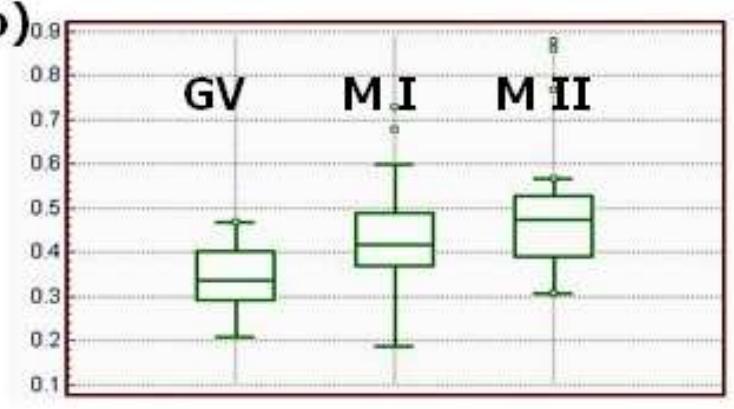

Figure 2. Experimental results with transmission spectra. (a) Average spectra for the three maturity stages. (b) Bow and Whiskers diagram [24].

Second, we measured the transmission spectra of individual oocytes. Figure 3 shows the transmission spectra of a single oocyte at three points in its maturation evolution, and is typical of the behavior of other oocytes we observed. The time interval between GV and MI is 5 hours compared to 16 hours between MI and MII. The time $\mathrm{T}=0$ refers to the first observation of the oocyte. The vertical arrow is a schematic representation of the possible evolution of the oocyte in terms of minimum transmission and wavelength during its maturation process. It seems that the measurement of the transmission spectra of the oocytes could be used to follow the maturation of the oocyte even in the 
intermediate stage between GV and MI or between MI and MII, despite the fact that visually, only these three stages can be distinguished. We will come back to this aspect in section four.

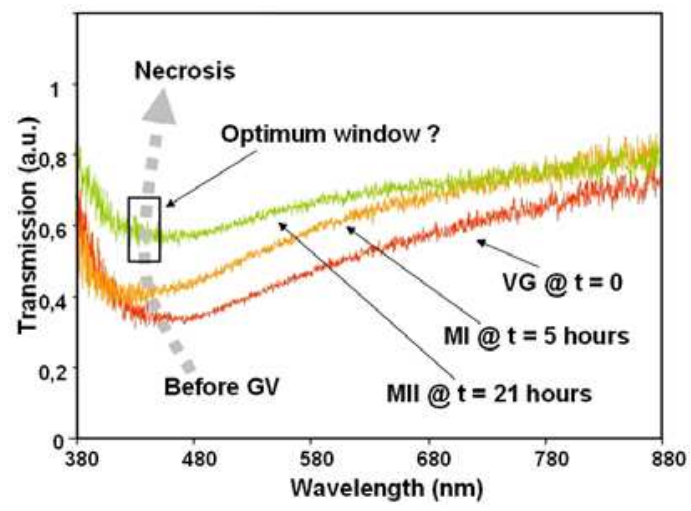

Figure 3. Temporal evolution of the transmission spectrum for a single oocyte [24].

\subsection{Refractive index measurement}

At this point, a question should be asked. Is the evolution of the transmission spectra only due to changes in absorption or could it be due to changes in the refractive index of the oocytes?

To answer this question, the absorption of the oocytes was estimated by means of image processing [22]. This is demonstrated in figure 4. Figure 4(a) shows an oocyte. The idea is to separate the part of the image relating to the oocyte from the background. To this end, a semi-automatic thresholding technique is used. The result is shown in figure 4(b). Once the oocyte is isolated, the absorption is obtained by dividing the average grey level of the oocyte by the average grey level of the background. The absorption was relatively constant, at $6 \%$, and did not differ greatly between oocytes.
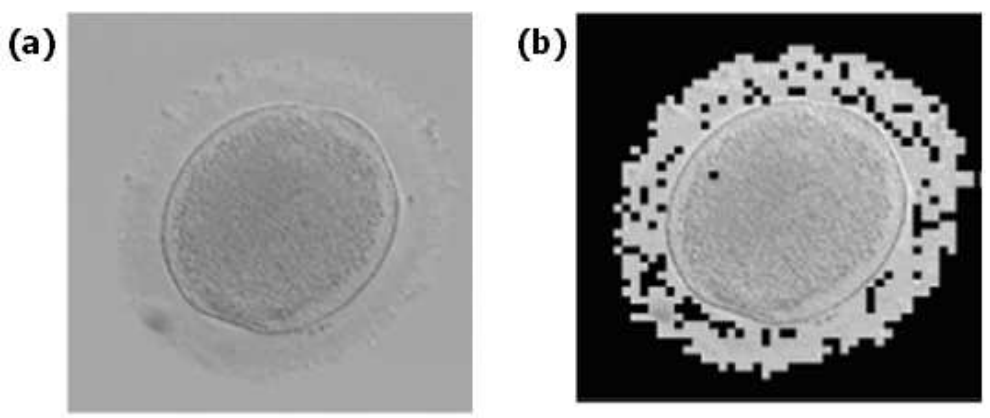

Figure 4. Image processing used to estimate the absorption of the oocytes. (a) The initial image. (b) Result of image processing when the oocyte is isolated [22].

Here we summarize the study described in detail in [22]. The principle is to place the oocyte between the two optical fibers of the microsystem shown in figure 1. The idea is to analyze the optical intensity collected for different oocyte positions between the fibers. Figure 5(a) explains the principle. When the oocyte is positioned close to the illumination fiber, a large amount of light is collected due to the oocyte that plays the role of a micro lens. Conversely, when the oocyte is positioned close to the collection fiber, the amount of light collected is much lower. The maximum collected light is obtained for a position between the two fibers.

The method we used consists in measuring the intensity for three positions of the oocyte: in contact with the illumination fiber, right in the middle of the fibers and in contact with the collection fiber. In this way, three experimental values are obtained as shown in figure 5(b). A computing model is then 
employed in order to fit theoretical data with experimental values. A MATLAB ${ }^{\circledR}$ program is used to compute the intensity collected as a function of the refractive index and for the three above-mentioned positions of the oocyte. Iteration by iteration, a value of the refractive index " $n$ " is found when the average distance between the experimental values and the computed ones is minimal (least mean square criterion). In this MATLAB ${ }^{\circledR}$ program, the oocyte is defined by a spherical core (cytoplasm) of refractive index $n_{c}$ surrounded by an envelope of refractive index $n_{p}$ (zona pellucida).

(a)
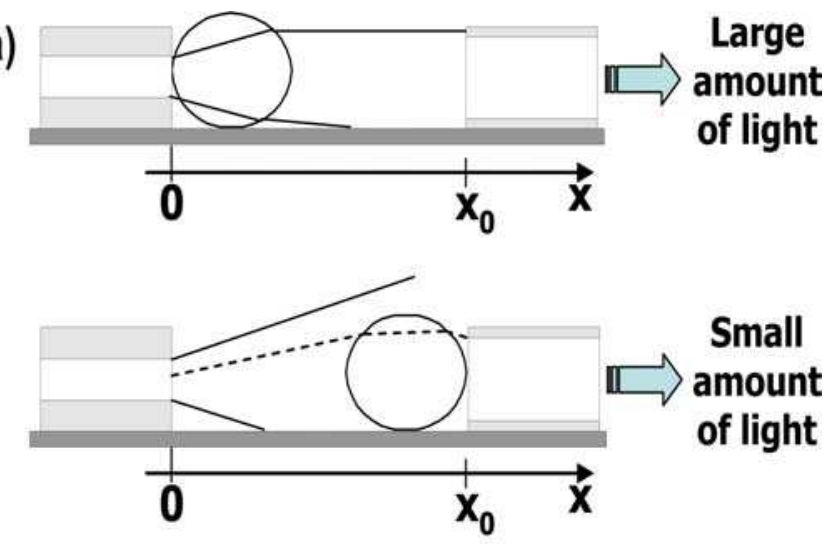

(b)

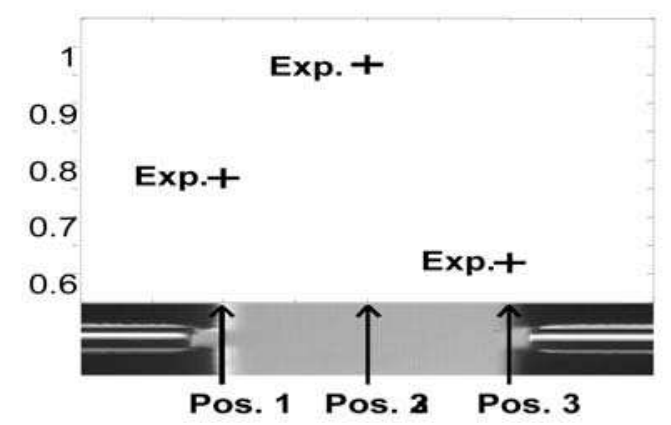

Figure 5. Method for measuring the refractive index of the oocytes [22].

Two types of measurements were considered. First, we calculated the average refractive indices in each stage of maturity. The results are presented in table 1 .

Table 1. Average refractive index of the oocyte for the three maturation stages [22].

\begin{tabular}{ccc}
\hline Stage & Average $\mathrm{n}_{\mathrm{c}}$ & $\begin{array}{c}\text { Average } \\
\mathrm{n}_{\mathrm{p}}\end{array}$ \\
\hline VG & $1.79^{ \pm 0.05}$ & $2.18^{ \pm 0.02}$ \\
M I & $1.86^{ \pm 0.06}$ & $2.10^{ \pm 0.02}$ \\
M II & $1.88^{ \pm 0.05}$ & $2.21^{ \pm 0.02}$ \\
\hline
\end{tabular}

We can see that the refractive index of the cytoplasm increases with maturity but nothing can be said about the refractive index of the zona pellucida. Furthermore, if we consider the values of the standard deviations, we may assume that although the three maturation stages can be observed individually, it seems impossible to use this optical technique to qualify the maturity of an unknown oocyte. 
We also considered the temporal evolution of the refractive indices for several individual oocytes. Results are presented in tables 2 and 3. Table 2 shows the results for the refractive index of the cytoplasm while table 3 shows the results for the zona pellucida.

Table 2. Temporal evolution of the refractive index of the cytoplasm [22].

\begin{tabular}{lccc}
\hline & $\begin{array}{c}\mathrm{N}_{\mathrm{c}} \text { at } \\
\mathrm{VG}\end{array}$ & $\begin{array}{c}\mathrm{N}_{\mathrm{c}} \text { at } \\
\mathrm{MI}\end{array}$ & $\begin{array}{c}\mathrm{N}_{\mathrm{c}} \text { at } \\
\mathrm{MII}\end{array}$ \\
\hline oocyte $\# 1$ & 1.78 & 1.79 & 1.91 \\
oocyte $\# 2$ & 1.83 & 1.93 & - \\
oocyte $\# 3$ & 1.74 & 1.84 & 1.89 \\
oocyte $\# 4$ & 1.745 & 1.85 & 1.89 \\
oocyte $\# 5$ & 1.70 & 1.84 & 1.86 \\
oocyte $\# 6$ & 1.84 & 1.79 & 1.79 \\
oocyte $\# 7$ & 1.78 & 1.85 & 1.88 \\
oocyte $\# 8$ & 1.83 & 1.84 & 1.87 \\
oocyte $\# 9$ & 1.86 & 1.85 & - \\
oocyte $\# 10$ & 1.88 & 1.915 & 1.924 \\
\hline
\end{tabular}

Table 3. Temporal evolution of the refractive index of the zona pellucida [22].

\begin{tabular}{lccc}
\hline & $\begin{array}{c}\mathrm{n}_{\mathrm{p}} \text { at } \\
\text { VG }\end{array}$ & $\begin{array}{c}\mathrm{n}_{\mathrm{p}} \text { at } \\
\text { MI }\end{array}$ & $\begin{array}{c}\mathrm{n}_{\mathrm{p}} \text { at } \\
\text { MII }\end{array}$ \\
\hline oocyte \#1 & 1.95 & 1.81 & 2.26 \\
oocyte \#2 & 2.36 & 2.47 & - \\
oocyte \#3 & 1.99 & 2.03 & 2.4 \\
oocyte \#4 & 1.93 & 2.02 & 2.29 \\
oocyte \#5 & 1.82 & 2.20 & 2.13 \\
oocyte \#6 & 2.45 & 2.14 & 1.89 \\
oocyte \#7 & 1.94 & 2.07 & 2.24 \\
oocyte \#8 & 2.28 & 2.24 & 2.21 \\
oocyte \#9 & 2.42 & 2.22 & - \\
oocyte \#10 & 2.47 & 2.24 & 2.32 \\
\hline
\end{tabular}

Here, we note that the general tendency is an increase in the cytoplasm refractive index (except for oocytes \#6 and \#9). Again, the result for the zona pellucida is not very clear. It should also be noted that in some cases, the theoretical model did not produce coherent values. The reasons for this are not yet understood.

2.5. Comparison with observations with a Transmission Electron Microscopy (TEM). 
In the previous sections, we have seen that the optical properties of the oocytes evolve with their level of maturity. Further experiments are required in order to evaluate the independent contribution of the absorption and refractive index. However, regardless of its origin, the evolution of the optical properties is linked to intra-cellular changes during the maturation of the oocytes. This is presented in figure 6, which compares the evolution of transmission spectra with TEM images [23].
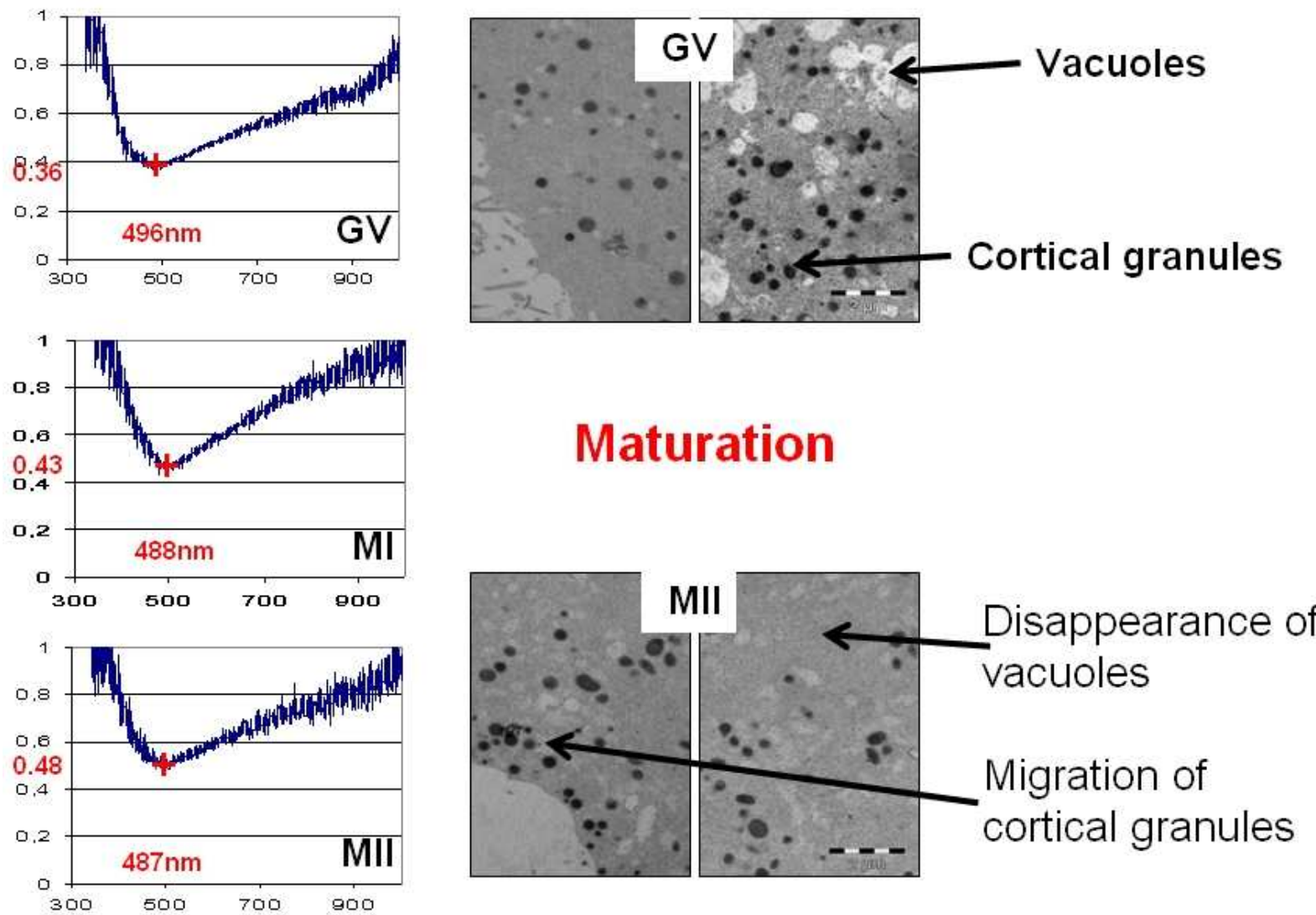

Figure 6. Relationship between optical properties and TEM examination [23].

It can be seen that the evolution of the spectra is concomitant with the migration of cortical granules toward the cortex and the reduction of vesicles and vacuoles from the center of the ooplasm. This also means that the intracytoplasmic evolution of the oocytes observed with a deleterious technique could be assessed with a simple and oocyte-compatible optical microsystem.

\section{Image processing}

\subsection{Short description of the Karhunen-Loeve Transform}

In this section, we summarize the results obtained using the Karhunen-Loeve Transform (KLT) applied to pictures of oocytes at different stages of maturity [17]. The KLT is a statistical technique based on the transformation of the data representation space. In the initial space, parameters describing an observation are correlated. The new space (composed of the principal components) is defined by the decomposition of the covariance matrix $(\mathrm{CM})$ in eigenvalues and eigenvectors. The principal axes of the new space are ordered in terms of importance [25, 26]. In this way, a description of the observation with uncorrelated parameters is obtained.

An important property of KLT is that, unlike the Fourier transform or the factorial analysis, the basis vectors are not known a priori but are "tailor made" to the given set of vectors. The first step is to take into account a set of $\mathrm{N}$ pictures to be analyzed. The KLT needs the calculation of the CM of the 
picture set after resorting of the picture pixels. To do this, we need to transform the initial square image matrices into vectors. The next step is to compute the eigenvalues of the CM and sort them in decreasing order. An eigenvector called the KL image is associated with each eigenvalue. The eigenvalue represents the percentage of information related to the corresponding KL image.

To illustrate the KLT, we can say that KL image 1 (eigenvector corresponding to the higher eigenvalue) is the mean picture. The differences between pictures are more obvious in the KL images related to small eigenvalues to be quantified [27].

\subsection{Processing protocol}

The chosen protocol depends on the types of oocytes we decided to investigate [17]. Here, we restrict the discussion to metaphase I and implemented metaphase II. The protocol consists in forming a "basis" with 12 pictures of a metaphase I oocyte whose level of maturity is obvious and cannot be doubted. We then computed the eigenvectors of the covariance matrix. The basic idea is to remove one picture of a metaphase I oocyte and replace it with another one. The next step is to analyze the variations of the eigenvalues associated with each picture replacement.

We decided to do this in two steps. Firstly, we replaced one MI with another MI (different from the 11 remaining MI in the initial set). This was done 12 times. We then computed the mean and rms of the variation of the eigenvalues. Secondly, we did the same with 12 pictures of implemented MII and we computed the mean and the rms again. The KL images that can separate the different stages of maturity correspond to those for which the difference between the two cases described above is maximized. We found channels 8 and 10.

\subsection{Experimental results}

To verify the ability of KLT to discriminate between the types of oocytes correctly, we plotted the eigenvalue attached to each oocyte replacement both types. The result is shown in figure 7 . We can clearly see that the two kinds of oocytes are separated. The "o" sign depicts the barycenters of the two sets and the two circles depict the limit of $1.4 \sigma$, where $\sigma$ is the dispersion. It involves a detection probability for a blind test of at least $75 \%$. The t-student test confirmed that the two stages were separated with an error probability of less than 0.001 . This means that image processing applied to oocyte pictures commonly taken in IVF centers can potentially discriminate between MI and implanted MII oocytes.

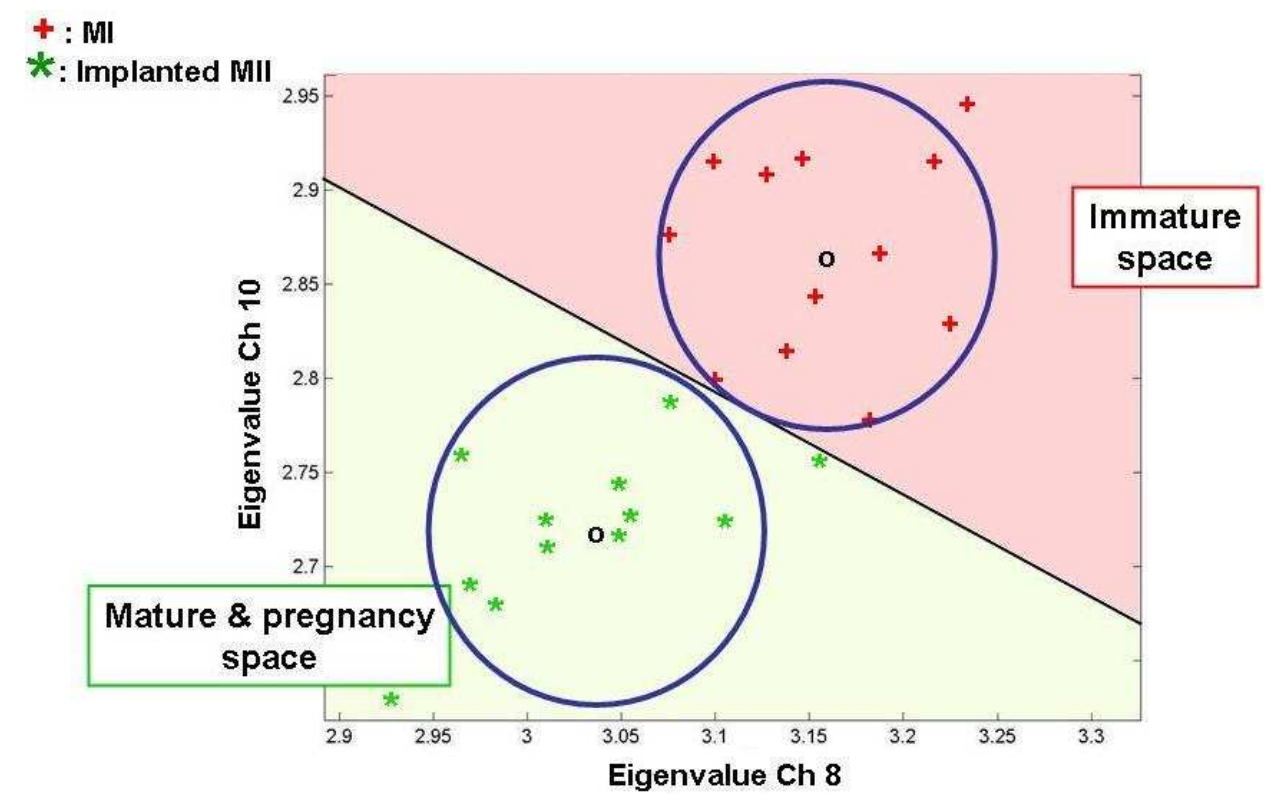


Figure 7. Eigenvalue $10 v s$. eigenvalue 8 for replacements with MI and implanted MII oocytes [17].

Blind tests were also conducted and the method was shown to be robust. Looking at figure 7, we can see that some oocytes are located near the boundary between the two half-spaces. We also see that some others are quite far from it. We have not yet applied KLT to a single oocyte during the maturation process but we expect that its location in the KLT space evolves during the maturation process.

\section{Short discussion}

As mentioned in section two, the three stages of maturity are easily identified by means of optical transmission properties. However, it seems difficult to define the maturation stage of a single oocyte precisely. This is unsurprising because oocytes are subject to a continuous maturation process from VG to MII. It is therefore unlikely that the optical properties discretely differ from one stage to another.

Figure 3 shows an arrow to describe this continuous evolution. We believe that an optimal temporal window exists that maximizes the probability of successful fertilization. The same arrow could be drawn on figure 7.

The three maturation stages exist, of course. They correspond to morphological characteristics that can be visually distinguished with a microscope [24]. Visible modifications, GV breakdown and first polar body extrusion, cannot entirely explain the changes in the spectrum. Through their volume, the changes in cytoplasm and zona pellucida organization in these final stages contribute to these optical modifications. Figure 6 illustrates this.

The boundary between two adjacent stages may be difficult to estimate visually, however. The qualification of oocytes in three stages, which are visually identified by various morphological characteristics, could be improved by analyzing certain oocyte properties in a more continuous manner together with the observation of the meiotic event, such as spindle formation and evolution, using a polarization microscope $[28,29]$. This complementary approach can therefore provide information on the modification of the zona pellucida during this maturation process [30].

These remarks are obviously open to further discussion. However, we believe that the oocytes should be chosen not only when they are MII but also at the right time during MII. We believe that the use of new and non-deleterious qualifying techniques that can assess the temporal evolution of the oocytes would be beneficial to IVF techniques.

Nowadays, micro technology in the field of optics and mechanics [31] coupled with image processing and other clinical and non-clinical qualification techniques should allow us to describe the continuous evolution of the maturity of the oocyte in a multidimensional space as illustrated in figure 8. We can therefore expect to be able to define an optimal trajectory in this multidimensional space. On the one hand, if the oocyte does not follow the right trajectory, we may conclude that it is not a good candidate for fertilization. On the other hand, if the oocyte evolves along the right trajectory, the biologist will wait for the oocyte to reach the right position in order to fertilize it. 


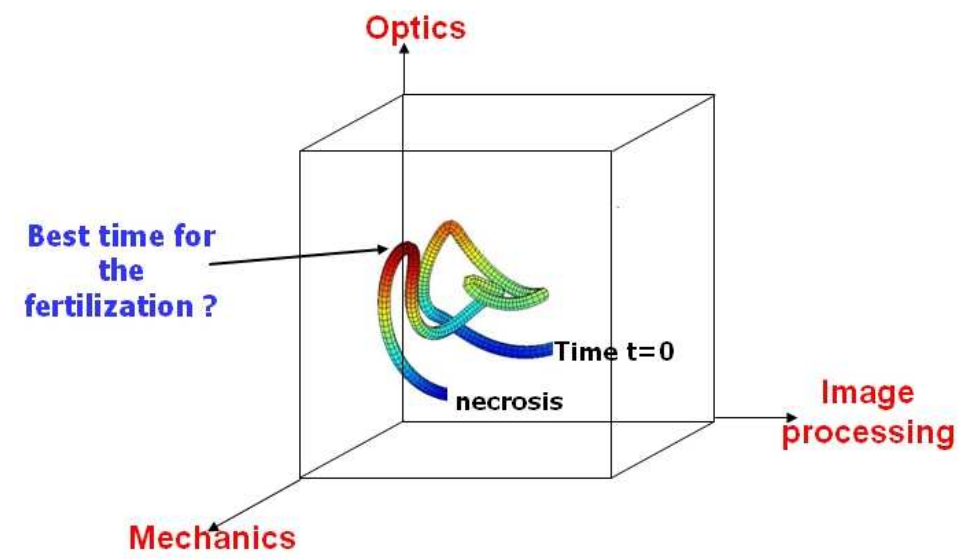

Figure 8. Example of a trajectory in a multidimensional space used to follow the maturation of oocytes.

\section{Conclusion}

In this paper, we have summarized some of our studies that aim to better qualify oocytes to be fertilized in medically assisted medicine.

We propose a simple optical microsystem used for measuring the optical transmission properties of oocytes. We showed that although the three stages of maturity are well identified, the device cannot be used in a blind test. However, the evolution of the transmission properties is concomitant with morphological changes in the oocyte cytoplasm. A better chance of a blind test is offered by image processing using Karhunen-Loeve Transform.

We believe it is unsurprising that there are difficulties in distinguishing between well separated stages. Indeed, oocytes undergo a continuous evolution process that can only be assessed with advanced and non-deleterious systems. Oocytes should therefore be chosen when they are MII but, more specifically, at the right time when they are MII. We thus provide a multidimensional representation of the maturation of the oocytes. The determination of an optimum trajectory in this space together with the determination of an optimum temporal window for fertilization should help increase the percentage of success in in vitro fertilization.

\section{References}

[1] Suh R S, Phadke N, Ohl D A, et al. 2003 Rethinking gamete/embryo isolation and culture with microfluidics Human Reproduction Update 9 451-461

[2] Beebe D J, Wheeler M, Zeringue H C. et al. 2002 Microfluidic technology for assisted reproduction Theriogenology 57 125-135

[3] Zeringue H C, Beebe D J, Wheeler M B 2001 Removal of cumulus from mammalian zygotes using microfluidic techniques Biomed. Microdev. 3 219-224

[4] Gauthier G, Piat E 2002 An electromagnetic micromanipulation system for single cell manipulation J. Micromech. 2 87-119

[5] Sadani, Z, Wacogne B, Pieralli C, et al. 2005 Microsystems and microfluidic device for single oocyte transportation and trapping: toward the automation of in vitro fertilising Sens. and Act. A $121364-372$

[6] Zeggari R, Wacogne B, Pieralli C. et al. 2007 A full micro-fluidic system for single oocyte manipulation including an optical sensor for cell maturity estimation and fertilisation indication Sens. and Act. B 125 664-671

[7] Kricka L J, Faro I, Heyner S, et al. 1997 Micromachined analytical devices: microchips for semen testing J. Pharm. Biomed. Anal. 15 1443-1447

[8] Danielli J F 1952 Division of the flattened egg Nature 170 496-496 
[9] Ohtsubo M, Hiramoto Y 1985 Regional differences in mechanical properties of the cell surface in dividing echinoderm eggs Dev. Growth Differ. 27 371-383

[10] Sawai T, Yoneda M 1974 Wave of stiffness propagating along the surface of the newt egg during cleavage $J$. Cell Biol. 60 1-7

[11] Nakamura S, Hiramoto Y 1978 Mechanical properties of the cell surface in starfish eggs Dev. Growth Differ. 20 317-327

[12] Murayama Y, Omata S, Constantinou C E 2004 Micro-mechanical sensing platform for the characterization of the elastic properties of the ovum via uniaxial measurement $J$. Biomech. $3767-72$

[13] Inui H, Nakamura H, Mizuno J, et al. 2006 Development of ovum evaluation system for human assisted reproductive technology: verification of the effects and the safety Proceedings in Hum. Reprod. 1(Suppl 1) i161

[14] Wacogne B, Pieralli C, Roux C, et al. 2008 Measuring the mechanical behaviour of human oocytes with a very simple SU-8 micro-tool Biomedical Microdevices 10 411-419

[15] Shaked N T, Rinehart M T, Wax A 2009 Dual interference channel quantitative phase microscopy of live cell dynamics Opt. Lett. 34 767-769

[16] Marquet P, Rappaz B, Magistretti P J, et al. 2005 Digital holographic microscopy: a noninvasive contrast imaging technique allowing quantitative visualization of living cells with subwavelength axial accuracy Opt. Lett. 30 468-470

[17] Pieralli C, Wacogne B, André C, et al. 2009 Biological qualification of oocyte maturity with the use of the Karhunen-Loeve transform: computer aided decision for selecting best oocytes before fertilization In Proceedings of the 1st International Workshop on Medical Image Analysis and Description for Diagnosis Systems DOI: 10.5220/0001810900770084

[18] Beuchat A, Thévenaz P, Unser M, et al. 2008 Quatitative morphometrical characterization of human pronuclear zygotes Hum. Reprod. 23 1983-1992

[19] Thompson J 2007 Culture without the Petri dish Therigenelogy 67 16-20

[20] Shen A G, Peng J, Zhao Q H, et al. 2012 Accurate and noninvasive embryos screening during in vitro fertilization (IVF) assisted by Raman analysis of embryos culture medium Laser Physics Letters 9 322-328

[21] Zeggari R, Wacogne B, Pieralli C 2006 Optics and microsystems for in vitro fertilisation Laser Physics 16 294-302

[22] Ivascu I, Zeggari R, Pieralli C, et al. 2010 Measuring the refractive index of oocytes by means of an optical microsystem : a non invasive technique to estimate the evolution of oocytes maturity In Proceedings of the Third International Conference on Biomedical Electronics and Devices DOI: 10.5220/0002756201270132

[23] André J, Amiot C, Vidberg F, et al. 2010 Measuring the oocyte cytoplasmic maturation process with a white light optical microsystem Proceedings in $J$. of Reproductive Medecine and Endocrinology 7284

[24] Vidberg F, Zeggari R, Pieralli C, et al. 2011 Measurement of oocyte temporal maturation process by means of a simple optical micro-system Sensors and Actuators B 157 19-25

[25] Chien Y T, Fu K S 1967, On the generalized Karhunen-Loeve expansion, IEEE trans. Information Theory, Vol. 13, pp. 518-520.

[26] Fukunaga, K., Koontz, W., L., G., 1970 Application of the Karhunen-Loeve expansion to feature selection and ordering IEEE Trans. Comp. C-19 311-318

[27] Comas L, Berthout P, Sabbah R, et al. 2005 Use of a 4-D cardiac phantom to quantify Karhunen-Loeve images applied to myocardial gated SPECT Comp. In Cardiology 32 541544

[28] Zeng H T, Ren Z, Yeung W S, et al. 2007 Low mitochondrial DNA and ATP contents contribute to the absence of birefringent spindle imaged with PolScope in in vitro matured human oocytes Hum. Reprod. 6 1681-1686 
[29] Montag M, van der Ven H 2008 Innovative techniques in human embryo viability assessment. Oocyte assessment and embryo viability prediction: birefringence imaging Reprod. Biomed. Online 4 454-460.

[30] Montag M, Schimming T, Köster M, et al. 2008 Oocyte zona birefringence intensity is associated with embryonic implantation potential in ICSI cycles Reprod. Biomed. Online 2 239-244

[31] Abadie J, Piat E, Oster S, et al. 2011 Modeling and experimentation of a passive low frequency nanoforce sensor based on diamagnetic levitation Sensors and Actuators A DOI : 10.1016/j.sna.2011.09.025 